\section{Plasma cystatin C correlates with GFR in patients with anorexia nervosa}

Renal disease occurs in over $70 \%$ of patients with anorexia nervosa. Detection of chronic kidney disease (CKD) in this group is, however, difficult as serum creatinine measurements are affected by the reduced muscle mass. Delanaye et al. investigated the value of cystatin $\mathrm{C}$ as an alternative marker of renal failure in patients with anorexia nervosa.

The researchers recruited 27 patients with anorexia nervosa (mean age $30 \pm 13$ years, 25 women) who had a BMI $<18 \mathrm{~kg} / \mathrm{m}^{2}$ (mean $15 \pm 2 \mathrm{~kg} / \mathrm{m}^{2}$ ). Glomerular filtration rate (GFR) was measured in all patients using ${ }^{51} \mathrm{Cr}$-ethylenediaminetetraacetate clearance (mean $68 \pm 23 \mathrm{ml} / \mathrm{min}$ ); 9 were classified as having stage $3 \mathrm{CKD}\left(\right.$ GFR $\left.<60 \mathrm{ml} / \mathrm{min} / 1.73 \mathrm{~m}^{2}\right)$ and 16 were diagnosed with stage 2 CKD (GFR 60$90 \mathrm{ml} / \mathrm{min} / 1.73 \mathrm{~m}^{2}$ ). Serum creatinine concentration was not notably correlated with GFR; however, the reciprocal of the plasma cystatin $C$ level showed a strong correlation with GFR (coefficient of correlation $r=0.62 ; P<0.001$ ). Detection of decreased kidney function (defined as GFR $<60 \mathrm{ml} / \mathrm{min} / 1.73 \mathrm{~m}^{2}$ ) was achieved with greater sensitivity and specificity by use of plasma cystatin $\mathrm{C}$ measurements $(67 \%$ and $100 \%$, respectively, at the optimal cut-off point) than by serum creatinine level $(56 \%$ and $78 \%$, respectively, at the optimal cut-off point).

Plasma cystatin $\mathrm{C}$ concentrations are not influenced by muscle mass, which explains why cystatin $C$ seems to be a better marker of renal function than is serum creatinine in patients with anorexia nervosa. The authors recommend confirmation of these results in a larger study population.

Original article Delanaye P et al. (2008) Cystatin C or creatinine for detection of stage 3 chronic kidney disease in anorexia nervosa. Nephron Clin Pract 110: c158-c163

\section{Hyperuricemia is an independent predictor of kidney disease}

Epidemiological studies have previously linked high blood uric acid levels to kidney disease. Now, an Austrian study has found that hyperuricemia is an independent predictor of kidney disease.
The study population comprised 21,475 healthy volunteers from the Vienna Health Screening Project who were followed up for a median of 7 years. Obermayr et al. classified participants into three groups based on their blood uric acid concentration: a 'reference' group of 19,466 people with uric acid levels $<416 \mu \mathrm{mol} / \mathrm{l}$; a 'slightly elevated' group of 1,821 people with uric acid levels of $416-529 \mu \mathrm{mol} / /$; and an 'elevated' group of 188 people with uric acid levels $\geq 535 \mu \mathrm{mol} / \mathrm{l}$. After adjustment for other risk factors, individuals in the 'slightly elevated' group and those in the 'elevated' group had odds ratios of 1.26 and 1.63 , respectively, for the development of stage 3 chronic kidney disease compared with the reference group. The influence of uric acid levels on the risk of incident kidney disease was greater in women than in men, and was substantially increased by hypertension. Odds ratios for new-onset kidney disease increased rapidly with increasing uric acid levels $(>357-416 \mu \mathrm{mol} / \mathrm{l}$ in women and $>416-476 \mu \mathrm{mol} / \mathrm{l}$ in men).

The authors conclude that prescription of uric acid-lowering drugs may be justified in apparently healthy hyperuricemic individuals, especially those with hypertension or prehypertension.

Original article Obermayr RP et al. (2008) Elevated uric acid increases the risk for kidney disease. J Am Soc Nephrol 19: 2407-2413

\section{Hormonal therapies can preserve fertility in patients receiving cyclophosphamide}

A recent study indicates that men and women with glomerulonephritis might be protected against cyclophosphamide-induced gonadal damage by testosterone or triptorelin (a gonadotropin-releasing hormone analog), respectively.

The study included 11 men and 17 premenopausal women treated with cyclophosphamide and prednisone. Male patients (mean age 32 years) received testosterone $(250 \mathrm{mg}$ intramuscularly every 15 days) throughout immunosuppressive therapy. Female patients (mean age 30 years) were divided into two groups: 13 received triptorelin during immunosuppressive therapy, and 4 received cyclophosphamide and prednisone only. Average treatment duration was 7 months for men 